\title{
Anatomía sistemática de la familia Anacardiaceae en México. I. La corteza de Tapirira Aublet.
}

\author{
TERESA TERRAZAS \\ Programa de Botánica, IRN, Colegio de Postgraduados. Montecillo, Edo. de México, 56230, México
}

\begin{abstract}
Resumen. La corteza de Tapirira se describe para las especies presentes en México. Los resultados muestran que hay caracteres anatómicos con valor diagnóstico que ayudan a diferenciar a las especies. El tamaño de los radios (altura y anchura) y el diámetro de los canales radiales de la corteza al igual que los de la madera ayudan a distinguir T. chimalapana de T. mexicana. La peridermis ondulada, la felodermis esclerificada y el exudado de color amarillocafé en $T$. chimalapana la distinguen de $T$. mexicana, con peridermis lisa, súber esclerificado y exudado de color rojo. Ambas especies presentan un ritidoma laminar, siendo más conspicuo en T. mexicana. Estudios adicionales de las especies del grupo de T. guianensis son esenciales para apoyar el valor diagnóstico de los caracteres antes mencionados.

Palabras clave: Anacardiaceae, anatomía de la corteza, Tapirira chimalapana, Tapirira mexicana.

Abstract. Tapirira bark anatomy is described for the species distributed in Mexico. Results show that there are anatomical characters with diagnostic value that contribute to distinguish between species. Bark ray dimensions (height and width) and diameter of the bark radial canals distinguish $T$. chimalapana from T. mexicana as do wood ray dimensions and wood radial canals. Ondulate periderm, esclerified phelloderm and yellow-brownish exudate characterize T. chimalapana, while $T$. mexicana possesses straight periderm, esclerified phellem and reddish exudate. Both species show a laminated rhytidome, more conspicuous in $T$. mexicana. Further studies in the species of $T$. guianensis group are essential to support the diagnostic value of the aforementioned characters.
\end{abstract}

Key words: Anacardiaceae, bark anatomy, Tapirira chimalapana, Tapirira mexicana.

\section{INTRODUCCIÓN}

El estudio anatómico de la corteza de especies leñosas no ha tenido un desarrollo tan amplio como la caracterización del xilema secundario (Roth, 1981). No obstante los trabajos realizados hasta la fecha con anatomía de la corteza han probado que ésta puede ser una herramienta valiosa en los estudios sistemáticos (Alfonso y Richter, 1991; Archer y Van Wyk, 1993; Richter y Van Wyk, 1990; Roth, 1981; Trockenbrodt y Paramenwaran, 1986; Van Wyk, 1985). La anatomía de la corteza al igual que la anatomía del xilema secundario es más clara para definir grupos a nivel genérico o supragenérico (Archer y Van Wyk, 1993; Richter, 1981). Sin embargo, las pocas investigaciones que han empleado los datos anatómicos de la corteza a nivel específico (Trockenbrodt y Parameswaran, 1986 y más referencias ahí), muestran que este tejido también ayuda a distinguir especies o grupos de especies.

Esau $(1964,1979)$ y Roth (1981) señalan los elementos de la corteza que generalmente poseen valor diagnóstico. Entre los más comunes se encuentran longitud del elemento de tubo criboso, número de células acompañantes, tipo de áreas cribosas en la placa cribosa, tipo de radios, distribución del esclerénquima, arreglo de células y cavidades secretoras y, disposición y tipo de cristales; así como características de las células de la peridermis.
Los estudios de anatomía comparada de la corteza en la familia Anacardiaceae son escasos. Roth $(1968,1981)$ describió la anatomía de la cọrteza de cinco especies de Anacardiaceae distribuidas en Venezuela. Zahur (1959) estudió algunas características de los elementos de tubo criboso del floema secundario para otras cuatro especies de Anacardiaceae y Gibson (1981) describió la corteza de Pachycormus. Mientras que el estudio del desarrollo de los canales secretores en el floema secundario ha ocupado la atención de varios autores (Bhatt y Moham Ram, 1992; Fahn y Evert, 1974; den Outer y van Veenendal, 1986; Venkaiah y Shah, 1984).

Esta investigación forma parte de un trabajo encaminado hacia el entendimiento del género Tapirira Aublet Anacardiaceae- (Terrazas y Wendt, 1995; Wendt y Mitchell, 1995) y es una contribución al conocimiento de la anatomía comparada de la corteza de las especies de Tapirira presentes en México. Además intenta encontrar caracteres que apoyen su identidad y comparar las características de la corteza de Tapirira con las de otros miembros de la familia estudiados hasta la fecha.

\section{MATERIALES Y MÉTODOS}

Las muestras de la corteza de Tapirira, incluyendo cámbium y xilema secundario fueron colectadas de doce individuos, 
Cuadro 1. Localidad y número de individuos colectados para el estudio de la anatomía de la corteza de Tapirira.

\begin{tabular}{|c|c|c|c|}
\hline Taxa & Localidad & Muestra & $\begin{array}{c}\text { Ejemplar de } \\
\text { respaldo * }\end{array}$ \\
\hline \multirow[t]{3}{*}{ T. chimalapana } & Sta. María Chimalapa, Oaxaca & 1,2 & 5296 \\
\hline & Uxpanapa, Veracruz & 3 & 5302 \\
\hline & Cerro Azul-Escolapa, Oaxaca & 4 & 5340 \\
\hline \multirow[t]{7}{*}{ T. mexicana } & Sta. María Chimalapa, Oaxaca & 5,6 & 5294 \\
\hline & Laguna Escondida, Veracruz & 7 & 5315 \\
\hline & Monte Líbano, Chiapas & 8 & 5317 \\
\hline & Chilón, Chiapas & 9 & 5325 \\
\hline & El Pericón, Chiapas & 10 & 5326 \\
\hline & Santa Rita, Chiapas & 11 & 5328 \\
\hline & Champerico, Chiapas & 12 & 5338 \\
\hline
\end{tabular}

* Los números de colecta corresponden a la colección de T. Wendt et al.

en localidades de los estados de Chiapas, Oaxaca y Veracruz (Cuadro 1). En la localidad de Santa María Chimalapa, Oaxaca las especies ocurren simpátricamente. Todas las muestras se colectaron de árboles con un diámetro a la altura del pecho > $30 \mathrm{~cm}$ y a $130 \mathrm{~cm}$ del suelo (Terrazas, 1988; Terrazas y Wendt, 1995) y se fijaron en Craf III (Johansen, 1940).

En el laboratorio se prepararon tres cubos de aproximadamente un $\mathrm{cm}$ de lado, por individuo colectado. Estos fueron incluidos en parafina, seccionados con un microtomo giratorio a $10 \mu \mathrm{m}$ de grosor, teñidos con safranina-verde fijo y montados con resina de Abies religiosa. La disociación de la corteza fue realizada de acuerdo al método de Jeffrey (Berlyn y Miksche, 1976). La medición de los elementos se realizó con un micrómetro del ocular. Se midieron o cuantificaron 25 unidades por muestra para cada caracter anatómico. Se obtuvieron algunos parámetros de la estadística descriptiva (SAS, 1989: valores de la media, una desviación estándar, mínimo y máximo). La terminología empleada se basa principalmente en Roth (1981), sin embargo se siguen las modificaciones propuestas por Trockenbrodt (1990). Los dibujos se hicieron usando una cámara lucida en un microscopio óptico Zeiss.

\section{RESULTADOS}

El Cuadro 2 resume las diferencias cuantitativas y cualitativas encontradas entre $T$. chimalapana y $T$. mexicana y en las Figuras 1 a 14 se ilustran caracteres distintivos de la corteza de Tapirira. En el Cuadro 3 se anotan algunas características anatómicas de las especies de Tapirira estudiadas así como las reportadas por otros autores con objeto de comparar la información disponible con relación a la corteza de Anacardiaceae. A continuación se presenta la descripción de la corteza externa e interna para el género en México.

\section{Características externas}

En $T$. chimalapana la corteza externa es fisurada a escamosa con escamas pequeñas que tienden a ser rectangulares y organizadas en líneas verticales o ligeramente diagonales situadas entre las fisuras (Fig. 1). En la parte interna de las fisuras el color varía del café rojizo pálido al rojo amarillento (HUE 5YR 4/6 y HUE 5Y/8; Munsell, 1975), mientras que la parte externa es color olivo (HUE 5Y4/3). La corteza interna es laminosa de color rojo (HUE 10R5/6) con exudado de color blanco o crema que al oxidarse cambia al amarillo-café.

En $T$. mexicana la corteza joven se exfolia con escamas de tamaño variable y líneas marcadas donde se inician las fisuras cuando joven. La adulta está fisurada con escamas gruesas, éstas son de forma rectangular y de tamaño más regular (Fig. 2). En ambos estados el color es rojizo obscuro (HUE 5YR2.5/2). La corteza interna laminosa es de color rojo (HUE 7.5/8). El exudado naranja o rojizo pálido forma gotas, las cuales se oxidan y obscurecen en pocos minutos. El grosor total de la corteza varía en ambas especies de 4 a $23 \mathrm{~mm}$.

\section{Características anatómicas}

La corteza presenta una zonación de tejidos bien definidos: con floema secundario no colapsado y colapsado, peridermis y ritidoma (Figs. 3, 4).

\section{Floema secundario}

El floema no colapsado varía de 1.0 a $1.3 \mathrm{~mm}$ de grosor, mientras el floema colapsado tiende a ser más ancho (Cuadro 2). Ambos se componen de elementos de tubo criboso, células acompañantes, parénquima axial, radios, esclerénquima y canales secretores (Figs. 5, 6). 
CuADro 2: Características cuantitativas y cualitativas de la corteza de las especies de Tapirira distribuidas en México. Clave: $\mathrm{M}=$ media, sd = una desviación estándar, mín. = valor mínimo, máx. $=$ valor máximo, $*=\mu \mathrm{m},+++=$ presencia,$-=$ ausencia.

\begin{tabular}{|c|c|c|c|c|c|c|c|c|}
\hline \multirow[b]{2}{*}{ Carácter } & \multicolumn{4}{|c|}{ T. chimalapana } & \multicolumn{4}{|c|}{ T. mexicana } \\
\hline & M & sd & mín. & máx. & M & sd & mín. & máx. \\
\hline Grosor floema no colapsado* & 1175 & 132 & 945 & 1328 & 1180 & 153 & 980 & 1340 \\
\hline Grosor floema colapsado* & 6844 & 312 & 6502 & 7239 & 4504 & 679 & 3133 & 5287 \\
\hline Longitud elemento de tubo criboso* & 689 & 100 & 522 & 885 & 438 & 119 & 282 & 658 \\
\hline Diámetro tangencial tubo criboso* & 29 & 5 & 22 & 36 & 30 & 5 & 22 & 36 \\
\hline \multicolumn{9}{|l|}{ No. de áreas cribosas en placa } \\
\hline cribosa & 7 & 2 & 5 & 11 & 4 & 1 & 2 & 6 \\
\hline Longitud fibra* & 1543 & 302 & 932 & 2875 & 988 & 225 & 611 & 1426 \\
\hline Diámetro tangencial fibra* & 19 & 3 & 16 & 24 & 22 & 3 & 16 & 26 \\
\hline Grosor pared de fibra* & 8 & 1 & 6 & 11 & 9 & 2 & 6 & 12 \\
\hline Altura radio* & 391 & 167 & 180 & 713 & 163 & 54 & 91 & 282 \\
\hline Anchura radio* & 41 & 24 & 16 & 102 & 24 & 4 & 20 & 28 \\
\hline Series radios & 2 & 0.4 & 1 & 3 & 3 & 0.3 & 2 & 3 \\
\hline \multicolumn{9}{|l|}{ Diámetro tangencial células procumbentes } \\
\hline de radio* & 12 & 2 & 10 & 14 & 11 & 2 & 8 & 15 \\
\hline No. de células entre radios & 6 & 2 & 3 & 9 & 7 & 3 & 2 & 14 \\
\hline Diámetro tangencial del canal radial* & 63 & 32 & 34 & 121 & 46 & 9 & 30 & 60 \\
\hline Diámetro canal longitudinal* & 33 & 11 & 24 & 94 & 79 & 14 & 60 & 103 \\
\hline \multicolumn{9}{|l|}{ No. de células epiteliales del canal } \\
\hline longitudinal & 22 & 1 & 21 & 24 & 9 & 2 & 8 & 11 \\
\hline Grosor peridermis* & 527 & 17 & 321 & 799 & 321 & 38 & 227 & 360 \\
\hline No. de células súber no obliteradas & 6 & 3 & 3 & 9 & 12 & 3 & 8 & 16 \\
\hline Grosor súber* & 323 & 70 & 242 & 504 & 170 & 10 & 157 & 186 \\
\hline No. de células felodermis & 6 & 1 & 4 & 8 & 8 & 1 & 6 & 10 \\
\hline Grosor felodermis* & 135 & 28 & 91 & 161 & 140 & 52 & 113 & 192 \\
\hline Cristales & & & +++ & & & & +++ & \\
\hline Taninos & & & +++ & & & & +++ & \\
\hline Súber con paredes gruesas & & & - & & & & +++ & \\
\hline Felodermis con paredes gruesas & & & +++ & & & & - & \\
\hline Peridermis ondulada & & & +++ & & & & - & \\
\hline Ritidoma fuertemente estratificado & & & - & & & & +++ & \\
\hline
\end{tabular}

CUADRo 3. Características anatómicas de la corteza de las especies de Tapirira estudiadas y de otras especies de Anacardiaceae (tomadas de Gibson (1981), Roth (1981) y Zahur (1959)). Especies: Tagu = Tapirira guianensis, Tame $=T$. mexicana, Tach $=T$. chimalapana, Angi $=$ Anacardium giganteum, Asle $=$ Astronium lecointei, Losa $=$ Loxopterygium sagotii, Padi $=$ Pachycormus discolor, Spmo $=$ Spondias mombin .

\begin{tabular}{|c|c|c|c|c|c|c|c|c|}
\hline \multirow[b]{2}{*}{ Carácter } & \multicolumn{8}{|c|}{ Especies } \\
\hline & Tagu & Tame & Tach & Angi & Asle & Losa & Padi & Spmo \\
\hline \multicolumn{9}{|l|}{ Esclerénquima } \\
\hline (fibras + , fibras y esclereidas \pm ) & $\mathrm{P}+$ & $\mathrm{P} \pm$ & $\mathrm{P} \pm$ & $\mathrm{P} \pm$ & $\mathrm{P} \pm$ & A & A & $\mathrm{P} \pm$ \\
\hline No. de series en radio & $1-3$ & $1-3$ & $1-3$ & $1-2$ & $1-3$ & $1-4$ & ? & $1-6$ \\
\hline Distancia entre radios (no. de células) & $2-10$ & $2-14$ & $3-9$ & $2-5$ & $5-15$ & $2-10$ & ? & $4-20$ \\
\hline Cristales & A & $\mathrm{P}$ & $\mathrm{P}$ & A & A & A & A & A \\
\hline No. de peridermis & $2-3$ & $2-3$ & $2-3$ & 1 & 1 & $10-15$ & 3 & $6-8$ \\
\hline Ritidoma escamoso & $\mathrm{P}$ & $\mathrm{P}$ & $\mathrm{P}$ & A & A & $\mathrm{P}$ & A & $\mathrm{P}$ \\
\hline Súber con engrosamientos & A & $\mathrm{P}$ & A & $\mathrm{P}$ & A & A & A & A \\
\hline Felodermis con engrosamientos & $\mathrm{P}$ & A & $\mathrm{P}$ & $\mathrm{P}$ & $\mathrm{P}$ & $P$ & A & $\mathrm{P}$ \\
\hline Color del exudado & a & b & $\mathrm{d} / \mathrm{a}$ & c & c & a & d & $\mathrm{c}$ \\
\hline
\end{tabular}

$\mathrm{P}=$ presencia, $\mathrm{A}=$ ausencia, ? = datos no presentados, $\mathrm{a}=$ amarillo, $\mathrm{b}=$ rojo, $\mathrm{c}=$ incoloro, $\mathrm{d}=$ blanco 
Elementos de tubo criboso. Los elementos de tubo criboso y células acompañantes se disponen en grupos de 4 a 20 células en $T$. chimalapana y de 3 a 14 en $T$. mexicana. Generalmente se arreglan en bandas tangenciales entre bandas de parénquima axial, ambas atravesadas por los radios de curso más o menos recto (Fig. 5). Los elementos de tubo criboso son células de pared delgada, de forma redonda o angulosa con un diámetro tangencial de $30 \pm 5 \mu \mathrm{m}$ para ambas especies $\mathrm{y}$, una longitud de $689 \pm 100 \mu \mathrm{m}$ en $T$. chimalapana y de $438 \pm 119 \mu \mathrm{m}$ en $T$. mexicana. La placa cribosa compuesta se dispone oblicuamente con 5 a 11 áreas cribosas. En el floema no colapsado de $T$. chimalapana se localizan de 5 a 7 bandas de tubos cribosos y células acompañantes, mientras que en $T$. mexicana 3 a 5 bandas.

Parénquima axial. El parénquima axial se arregla en forma distintiva en ambas especies. En $T$. chimalapana forma bandas tangenciales de 1 a 4 células de grosor entre las bandas de tubos cribosos y células acompañantes (Fig. 6). A medida que el floema no colapsado es más cercano a la peridermis, se aprecia un aumento en taninos y cristales. En $T$. mexicana el parénquima axial no forma bandas regulares y toma una disposición alada con respecto a los canales secretores. Esta disposición lo hace muy conspicuo como consecuencia del aumento de taninos en el floema colapsado (Fig. 7).

Esclerénquima. El esclerénquima se arregla en bandas con 4 a 5 células de grosor, desde el cámbium hasta la peridermis y se aprecia, además, en algunas regiones del ritidoma (Figs. 3, 4, 6). Las bandas de esclerénquima son atravesadas por los radios que se esclerifican progresivamente (Fig. 8), contribuyendo a formar bandas casi continuas cerca de la peridermis (Fig. 12). El esclerénquima está constituido por fibras y esclereidas, éstas ubicadas a ambas caras de la banda (Fig. 9). Las fibras dispuestas en la parte central de la banda son de lumen pequeño (Fig. 12), con un grosor de pared de $8 \pm 2 \mu \mathrm{m}$ y un diámetro tangencial de $20 \pm 3$ $\mu \mathrm{m}$. Su longitud varía de $988 \pm 225 \mu \mathrm{m}$ en $T$. mexicana a $1543 \pm 302 \mu \mathrm{m}$ en $T$. chimalapana. Las fibras presentan punteaduras conspicuas que pueden ser escasas o moderadamente abundantes. La abundancia de cristales romboédricos en las esclereidas aumenta notablemente hacia la parte externa del floema colapsado (Fig. 9). En esta región se distinguen esclereidas del sistema vertical fuertemente engrosadas en T. chimalapana (Fig. 12). Estas deforman la banda y comúnmente contribuyen a darle continuidad. Mientras que en $T$. mexicana algunas esclereidas presentan muy ligeros engrosamientos tangenciales o radiales. En esta especie la dilatación, la abundancia de canales secretores y la disposición de las bandas del esclerénquima en forma escalonada ocasionan que casi nunca se formen bandas continuas (Fig. 4).

Radios. Los radios son heterocelulares uni y multiseriados. Los uniseriados son escasos, predominantemente hay biseriados en $T$. chimalapana y triseriados en $T$. mexicana (Figs. 10,11). Las células procumbentes son de 8 a $15 \mu \mathrm{m}$ de diámetro tangencial y constituyen el cuerpo del radio. Las células erectas de los radios pueden variar en número de 1 a 3 células en cada extremo. Algunos radios son fusionados, especialmente en T. mexicana. Los radios se disponen en el floema no colapsado a una distancia entre sí de $6 \pm 2$ células en $T$. chimalapana y de $7 \pm 3$ en $T$. mexicana. Su curso es recto en esta región antes de cruzar la primera banda de esclerénquima o ubicarse cerca de un canal secretor longitudinal. Las dimensiones de los radios difieren entre las especies. En $T$. chimalapana la anchura es de $41 \pm 24 \mu \mathrm{m}$ y la altura de $391 \pm 167 \mu \mathrm{m}$, y en T. mexicana la anchura es de $24 \pm 4 \mu \mathrm{m}$ y la altura de $163 \pm 54 \mu \mathrm{m}$. Algunos radios presentan canales secretores, siendo más abundantes en $T$. chimalapana (Fig. 8). Ocasionalmente las células contienen taninos y cristales romboédricos. En el floema colapsado se aprecia una ligera dilatación en algunos radios: de 2 a 3 series pasan a ser de 4-6 y aumenta el contenido de los cristales.

Canales secretores. Se presentan canales secretores longitudinales y radiales (Figs. 7, 8). Ambos se distribuyen irregularmente, sin embargo en la parte interna del floema colapsado de $T$. chimalapana la disposición de los canales longitudinales parece ser en hileras tangenciales y en $T$. mexicana son más abundantes y conspicuos debido a que el parénquima axial tiene una disposición alada. Los canales longitudinales son más abundantes en ambas especies que los radiales. En T. mexicana los canales radiales son raros. El diámetro tangencial de los canales secretores longitudinales en $T$. chimalapana es de $33 \pm 11 \mu \mathrm{m}$, mientras que en $T$. mexicana varía de $79 \pm 14 \mu \mathrm{m}$ en el floema no colapsado hasta $144 \pm 51 \mu \mathrm{m}$ en el ritidoma. El diámetro tangencial de los canales radiales en $T$. chimalapana es de $63 \pm 32 \mu \mathrm{m}$ y en T. mexicana de $46 \pm 9 \mu \mathrm{m}$. Ambos tipos están bordeados internamente por una capa de células epiteliales, generalmente con protoplasma. Una segunda o, a veces, una tercera capa de células parenquimatosas rodean el canal y ocasionalmente acumulan taninos (Figs. 7, 8). Estas células corresponderían a las células de la vaina del canal. El número de células epiteliales varía de 9 a 22 en los canales longitudinales.

Dilatación. El inicio de la dilatación se aprecia en algunos radios y en algunas células del parénquima axial a partir del floema no colapsado. La dilatación es ligera y esparcida.

Peridermis

La peridermis es ondulada irregulamente con respecto al cámbium en $T$. chimalapana (Fig. 3) y es casi paralela al cámbium u ocasionalmente presenta ligeras ondulaciones en $T$. mexicana (Fig. 4). La peridermis tiene un grosor de $527 \pm 17 \mu \mathrm{m}$ en $T$. chimalapana y de $321 \pm 38 \mu \mathrm{m}$ en $T$. mexicana.

En $T$. chimalapana la peridermis se desgaja para formar escamas que envuelven parte de esclerénquima y células obliteradas, así como canales secretores. Las células del súber de pared delgada (Fig. 13), obliteradas o no, presentan un arreglo en hileras radiales y dan al súber un tamaño medio (Roth, 1981) con un grosor de $323 \pm 70 \mu \mathrm{m}$. Las no obliteradas forman hileras de 3 a 13 células ensanchadas tangencialmente, con contenidos rojos en forma de gotas. Las obliteradas forman hileras de aproximadamente 
de 31 a 45 células. La felodermis se compone de 4 a 8 células isodiamétricas que le dan un grosor de $135 \pm 28 \mu \mathrm{m}$. La mayoría de las células contiene taninos de color café rojizo; algunas células portan cristales prismáticos. Las células más internas se esclerifican y pierden su disposición radial, confundiéndose con las células del floema (Fig. 13).

En $T$. mexicana las células del súber son ensanchadas tangencialmente y de pared gruesa, obliteradas o no, presentan una disposición radial (Fig. 14). Las no obliteradas forman hileras radiales de 8 a 16 células que contienen gotas de color rojizo. Las obliteradas en hileras inclinadas no se pueden contar. En conjunto, dan al súber un tamaño de $170 \pm 10 \mu \mathrm{m}$. La felodermis, de 6 a 10 células isodiamétricas de pared delgada con taninos y cristales, se encuentra en hileras radiales (Fig. 14). Esta tiene un grosor de $140 \pm 52 \mu \mathrm{m}$. Ritidoma

El ritidoma contiene de 2 a 3 peridermis (Figs. 3, 4), y forma una corteza de escamas exfoliantes pequeñas en $T$. chimalapana y de tamaño variable en $T$. mexicana. En general, en los desgajamientos de la peridermis en $T$. chimalapana se aprecian parte del esclerénquima y algunos canales secretores longitudinales, rodeados completamente de esclereidas y células obliteradas del floema. Estas no tienen un arreglo regular entre las peridermis. Por otro lado, en $T$. mexicana se pueden apreciar aproximadamente cuatro bandas de esclerénquima entre las peridermis (Fig. 4), asî comolos canales secretores longitudinales reducidos radialmente y rodeados de esclereidas y células obliteradas del floema. La disposición alterna de los elementos antes mencionados dan la apariencia de un ritidoma claramente estratificado.

\section{DISCUSIÓN}

Tapirira mexicana y $T$. chimalapana tienen una éstructura muy similar, sin embargo presentan diferencias cualitativas y cuantitativas que a continuación se discuten (Cuadro 2). Los elementos de tubo criboso se consideran para ambas especies del tipo II de Zahur (1959), pero difieren en longitud. Los elementos de tubo criboso de Tapirira son más largos que en los otros géneros estudiados por Zahur (1959) para la familia. Tapirira chimalapana forma grupos de tubos cribosos y células acompañantes funcionales de tamaño más regular y más conspicuos que $T$. mexicana. Esto posiblemente se deba a la disposición del parénquima axial en bandas tangenciales que separan los grupos regularmente. Mientras que el grosor del floema no colapsado fue muy similar para ambas especies, el grosor del floema colapsado difiere ampliamente. Estos valores sugieren una tasa diferencial de crecimiento, por lo que estudios de desarrollo serían muy importantes.

Lacontinuidad y el grosor de las bandas de esclerénquima difiere entre las especies. Tapirira chimalapana presenta bandas continuas cerca de la peridermis. La continuidad es dada principalmente por el engrosamiento marcado de algunas esclereidas radiales y verticales. Estas esclereidas corresponderían a las células pétreas que Roth (1968) describe para T. guianensis. En T. mexicana las bandas de esclerénquima son delgadas y generalmente superpuestas, y en pocos casos llegan a ser continuas. Roth (1981) y Zahur (1959) encontraron fibras y esclereidas en Astronium lecointei y Mangifera indica (Cuadro 3). No se encontró esclerénquima en especies de Loxopterygium, Rhus y.Pachycormus (Gibson, 1981; Roth, 1968; Solereder, 1908). La longitud promedio de las fibras difiere en las especies de Tapirira estudiadas. Esta característica junto con otras, podría ser de valor diagnóstico (Chattaway, 1959). Sin embargo, la longitud de las células floemáticas tiende a ser más corta en T. mexicana, lo que podría explicarse como una maduración más lenta en esta especie.

Los radios son del tipo I de Roth (1981). Estos presentaron diferencias cuantitativas entre ambas especies tanto en altura, anchura y distancia entre radios, por lo que podrían considerarse con valor diagnóstico. A las células de los radios que se esclerifican en el floema colapsado, Bamber (1962) las denominó radio-esclereidas y es el término que debe emplearse para distinguirlas de las otras células de los radios. Las radio-esclereidas son relativamente abundantes, hecho que difiere de lo encontrado por Roth (1968) para $T$. guianensis y otros géneros de la familia.

Los canales secretores longitudinales que distinguen la corteza de Anacardiaceae (Cronquist, 1981; Roth, 1981) se observaron en ambas especies estudiadas. Roth (1968) sólo señala la presencia de canales secretores longitudinales y radiales en Loxopteriygium sagotii, sin embargo ambos tipos se observaron en el material estudiado. Para T. chimalapana los canales radiales son de mayor diámetro y más abundantes que en T. mexicana. Con respecto a los canales longitudinales en T. mexicana, éstos aumentan su diámetro tangencial a medida que se acercan a la superficie de la corteza. El ensanchamiento puede deberse a la anastomosis de dos o más canales o a la dilatación (Gibson, 1981; Kucera y Butterfield, 1977). Es importante señalar que debido a la clara diferencia entre las células epiteliales, las células que rodean a las epiteliales y las procumbentes, se denominó a las segundas, células de la vaina del canal de acuerdo con Werker y Fahn (1969).

Los resultados sugieren que el exudado de la corteza en las especies de Tapirira proviene de los canales longitudinales y radiales, por lo que no debe denominarse 'látex' al exudado de Tapirira. La palabra látex indica que esta emulsión, con diferente composición química, se halla contenida en laticíferos (Font Quer 1977) y, éstos no se observaron en Tapirira, ni tampoco han sido observados en otras Anacardiaceae (Gibson, 1981; den Outer y van Veenendal, 1986; Roth, 1981; Venkaiah y Shah, 1984).

Con respecto a los cristales, Roth $(1968,1981)$ y Gibson (1981) no mencionan su presencia en los géneros estudiados, sin embargo se encontraron en ambas especies. Sería interesante verificar la ocurrencia de cristales en otros géneros de la familia.

La peridermis de T. chimalapana es ondulada (Roth, 1981). La ondulación tiene valor diagnóstico por su rara ocurrencia (Roth, 1981). Ambas especies presentan un súber 
de tamaño regular al igual que $T$. guianensis, pero en $T$. mexicana éste está esclerificado. La felodermis es de grosor regular (Roth, 1981) para ambas especies. En T. mexicana la felodermis no es esclerificada, a diferencia de T. chimalapana y $T$. guianensis, caracter que podría ser diagnóstico para las especies del género dentro del grupo de $T$. guianensis (Terrazas y Wendt, 1995).

El ritidoma de T. mexicana y $T$. chimalapana es semejante a T. guianensis (Roth, 1981). En las tres especies el ritidoma tiende a ser laminar o estratificado. Esta característica también es compartida por T. bethanniana (Mori y Mitchell, 1990; Terrazas, obs. pers.). La diferencia en engrosamiento de las células del súber puede ser uno de los factores por los que hay diferencias en tamaño y forma de las escamas.

Las características externas de la corteza ayudan en la identificación de los individuos adultos, principalmente por el tamaño de las escamas y por el color del exudado (Wendt y Mitchell, 1995).

Las características anatómicas encontradas por Roth $(1968,1981)$ y Zahur (1959) para la corteza de las Anacar- diaceae, en su mayoría fueron observadas en T. mexicana y T. chimalapana. El análisis de las dos especies estudiadas sugiere que los caracteres anatómico cuantitativos: longitud del elemento de tubo criboso, longitud de fibras, altura de radios, número de series en los radios, y algunos caracteres cualitativos de peridermis (felodermis y súber) y ritidoma, tienen valor diagnóstico. Es importante destacar que las diferencias encontradas entre las dos especies en relación con los caracteres cuantitativos de radios y canales radiales en la corteza también fueron encontrados para estas estructuras en el xilema secundario (Terrazas y Wendt, 1995). Por lo tanto, estudiar la anatomía de la corteza de $T$. bethanniana, $T$. obtusa y $T$. retusa es fundamental para apoyar el valor diagnóstico de los caracteres mencionados anteriormente.

Considerando el poco número de especies estudiadas para la familia Anacardiaceae es difícil sugerir que caracteres de la corteza podrían ser de utilidad en la sistemática de la familia, por lo que se recomienda continuar los estudios de este tejido en otros géneros de Anacardiaceae.
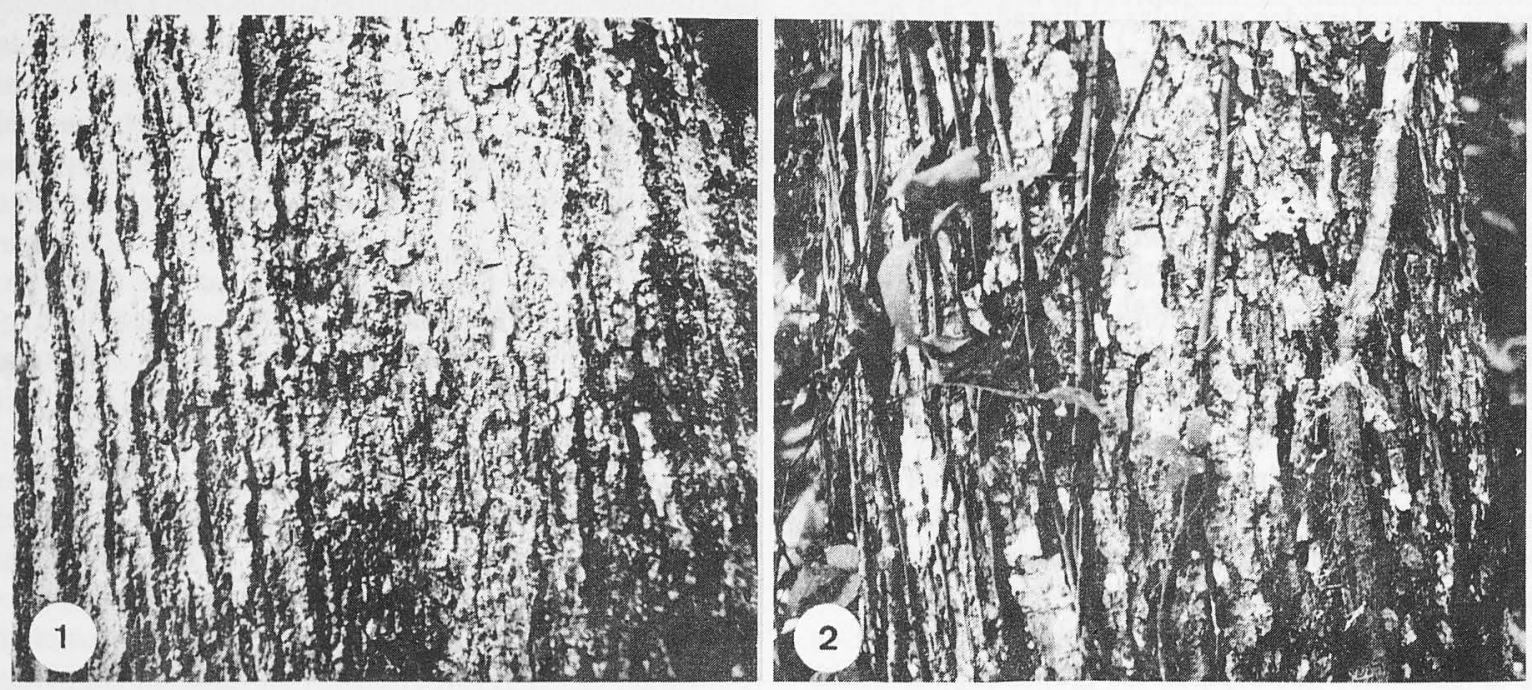

Figuras 1-2. Corteza de Tapirira. 1. T. chimalapana (4). 2. T. mexicana (10). El número en paréntesis corresponde al de la muestra en el Cuadro 1. 


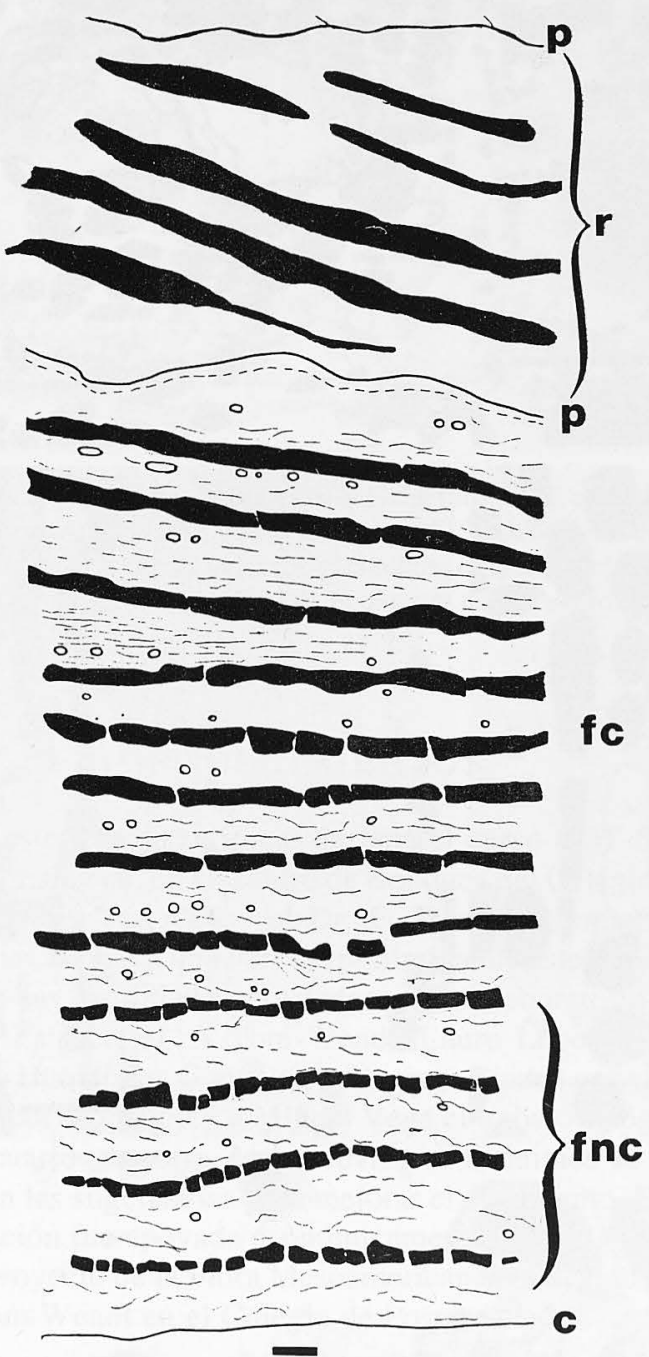

3
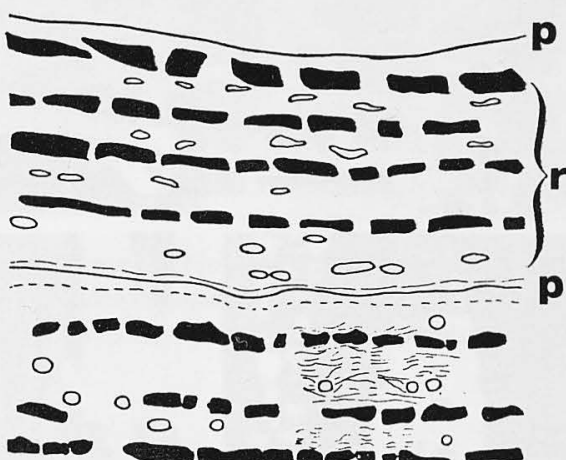

$\rightarrow \infty x^{2}$
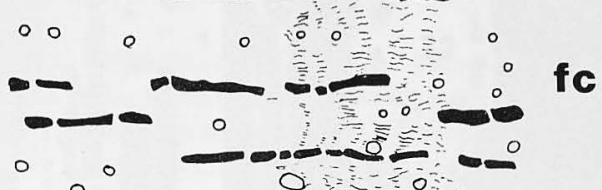

(1)

$10000=0$
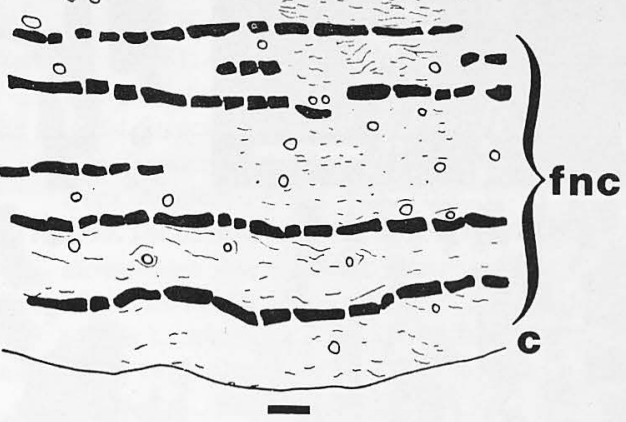

4

Figuras 3-4. Representación esquemática de la corteza en vista transversal. El esclerénquima se muestra en negro sólido, círculos vacios representan canales longitudinales y líneas finas taninos en parénquima axial. 3. T. chimalapana (3). 4. T. mexicana (5). Escala $=300 \mu \mathrm{m} . \mathrm{c}=$ cámbium vascular; $\mathrm{fnc}=$ floema no colapsado; $\mathrm{fc}=$ floema colapsado; $\mathrm{p}=$ peridermis; $\mathrm{r}=$ ritidoma. El número en paréntesis corresponde al de la muestra en el Cuadro 1. 


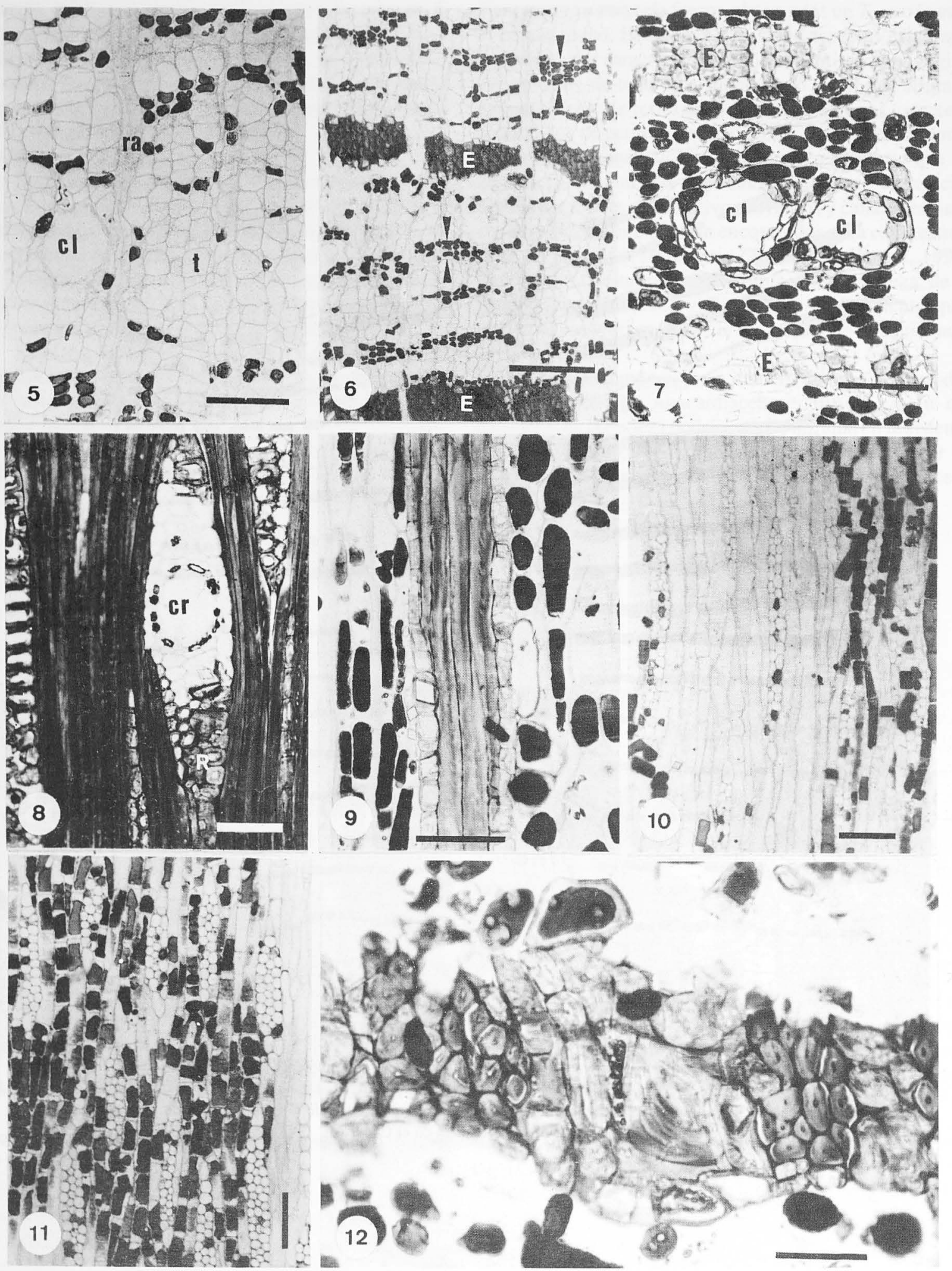

Figuras 5-12. Características del floema no colapsado y colapsado. 5 T. mexicana (5) T.Tubos cribosos y células acompañantes en el floema no colapsado; nótese un canal longitudinal jóven. 6. T chimalapana (2) T. Bandas de esclerénquima y parénquima axial con pocos taninos en floema no colapsado (flechas). 7. T. mexicana (6) T. Canales longitudinales y parénquima axial con abundantes taninos en floema colapsado. 8. T. chimalapana (2) LT. Canal radial y abundantes radio-esclereidas, nótese la esclerificación progresiva de los radios. 

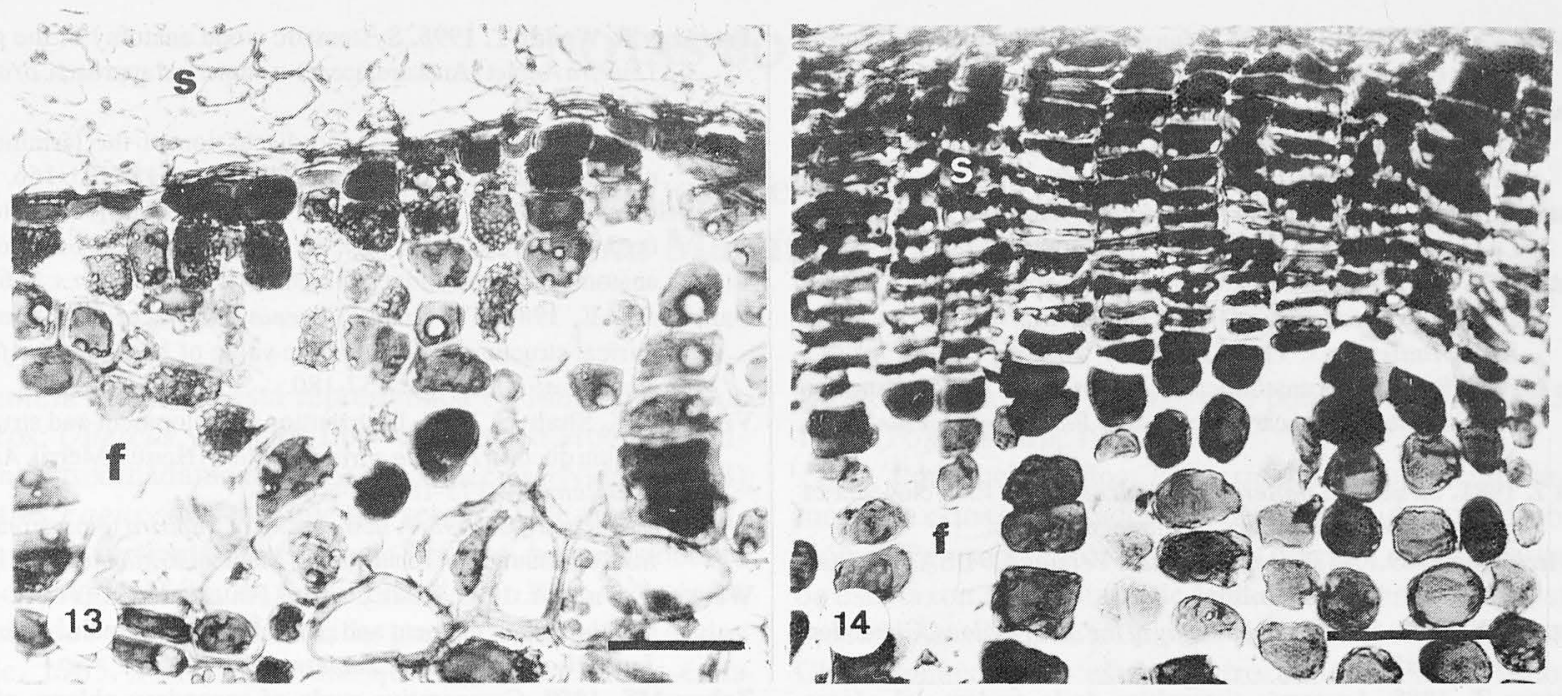

Figuras 13-14. Características de la peridermis. 13.T. chimalapana (4) T. Nótense las células de la felodermis con paredes engrosadas y delgadas en el súber. 14. T. mexicana (5) T. Las células del súber con paredes engrosadas y delgadas en la felodermis. Nótese la disposición en hileras radiales de las células en esta especie. Escala $=50 \mu \mathrm{m} . \mathrm{T}=$ corte transversal; $\mathrm{f}=$ felodermis; $\mathrm{s}=$ súber. El número en paréntesis corresponde al de la muestra en el Cuadro 1.

\section{AGRADECIMIENTOS}

Parte de este trabajo se inició durante el curso BOT-670, Problema Especial, en el Centro de Botánica del Colegio de Postgraduados. Agradezco al Dr. E. Mark Engleman las sugerencias hechas a una versión preliminar de este trabajo, así como las facilidades prestadas por el laboratorio de Botánica Estructural. A Tom Wendt, Lauro López Mata, Heriberto Hernández González y Santiago Sinaca su ayuda en la colecta de campo y a Miguel Vega el trabajo fotográfíco de cuarto obscuro. A dos revisores anónimos se les agradecen las sugerencias para mejorar el documento. Esta investigación fue apoyada económicamente por el CONACYT al proyecto de la Flora Mesoamericana conducido por el Dr. Tom Wendt en el Colegio de Postgraduados.

\section{LITERATURA CITADA}

Alfonso VA, Richter HG. 1991. Wood and bark anatomy of Buchenavi Eichl. (Combretaceae). IAWA Bulletin n.s. 12: 123-141.

Archer RH, Van Wyk AE. 1993. Bark structure and intergeneric relationships of some African Cassinoideae (Celastraceae). IAWA Journal 14: 35-53.

Bamber RK. 1962. The anatomy of the barks of Leptospermoideae. Australian Journal of Botany 10: 25-54.
Berlyn GP, Miksche JP. 1976. Botanical microtechnique and cytochemistry. Iowa State University Press, Ames.

Bhatt JR, Mohan Ram HY. 1992. Development and ultrastructure of primary secretory ducts in the stems of Semecarpus anacardium (Anacardiaceae). IAWA Bulletin n.s. 13: 173-185.

Chattaway MM. 1959. The anatomy of bark. VII. Species of Eugenia. Tropical Woods 111: 1-14.

Cronquist A. 1981. An integrated system of classification of flowering plants. Columbia University Press, New York.

Esau K. 1964. Structure and development of the bark in dicotlyledons. En: Zimmermann ML, edr. The formation of wood in forest trees. Academic Press, New York, 37-50.

Esau K. 1979. The phloem. En: Metcalfe CR, Chalk L. edrs. Anatomy of the dicotyledons. 2nd. ed. Vol. I. Clarendon Press, Oxford, 181-189.

Fahn A, Evert RF. 1974. Ultrastructure of the secretory ducts of Rhus glabra L. American Journal of Botany 61: 1-14.

Font Quer P. 1977. Diccionario de botánica. Sexta reimpresión, Ed. Labor, S.A., Barcelona, España.

Gibson AC. 1981. Vegetative anatomy of Pachycormus (Anacardiaceae). Botanical Journal of the Linnean Society 83: 273-284.

Johansen DE. 1940. Plant microtechnique. McGraw-Hill, New York.

Kucera LJ, Butterfield BG. 1977. Resin canals in the bark of Phyllocladus species indigenous to New Zealand. New Zealand Journal of Botany 15: 657-663.

Mori SA, Mitchell JD. 1990. Tapirira bethanniana (Anacardiaceae) and Eschweilera piresii subsp. viridipetala (Lecythidaceae). Memoirs of the New York Botanical Garden 64: 229-234.

9. T. mexicana (6) LR. Banda de esclerénquima con fibras, esclereidas y cristales romboédricos a ambos lados de las fibras. 10. T. chimalapana (2) LT. Radios biseriados. 11. T. mexicana (8) LT. Radios triseriados. 12. T. chimalapana (3) T. Esclereidas fuertemente engrosadas en floema colapsado. Escala: 5,6 y $8=200 \mu \mathrm{m} ; 7,9-12=100 \mu \mathrm{m}$. T = corte transversal; $\mathrm{LT}=$ corte tangencial $; \mathrm{LR}=$ corte radial. $\mathrm{cl}=$ canal longitudinal; $\mathrm{cr}=\mathrm{canal}$ radial; ra $=$ radio; $\mathrm{t}=$ elemento de tubo criboso; $\mathrm{E}=$ esclerénquima; $\mathrm{R}=$ radio-esclereida. El número en paréntesis corresponde al de la muestra en el Cuadro 1 . 
Munsell Color Company, 1975. Munsell soil color chart. Munsell Color Co., Maryland:

Outer RW den, van Veenendaal WLH. 1986. Distribution and development of secretory ducts in Trichoscypha (Anacardiaceae). Acta Botanica Neerlandica 35: 425-435.

Richter GH. 1981. Anatomie des sekundaren xylems und der rinde der Lauraceae. Verlag Paul Parey, Hamburg.

Richter HG, Van Wyk AE. 1990. Wood and bark anatomy of Lauraceae IV: Dahlgrenodendron J.H. Van der Merwe \& Van Wyk. IAWA Bulletin n.s. 11: 173-182.

Roth I. 1968. Estructuras anatómicas de la corteza de algunas especies venezolanas de Anacardiaceae. Acta Biológica Venezuelica 6: 146-159.

Roth I. 1981. Structural patterns of tropical barks. Encyclopedia of plant anatomy. Gebruder Borntraeger, Berlin.

SAS Institute.1989. SAS Procedures Guide Version 6.04. SAS Institute Inc., Cary, North Carolina.

Solereder H. 1908. Systematic anatomy of the dicotyledons. Clarendon Press, Oxford.

Terrazas T. 1988. Anatomía sistemática de la madera del género Tapirira (Anacardiaceae) con énfasis en México. Tesis Maestría, Colegio de Postragaduados, Chapingo, México.
Terrazas T, Wendt T. 1995. Systematic wood anatomy of the genus Tapirira Aublet (Anacardiaceae) -a numerical approach. Brittonia 71: 109-119.

Trockenbrodt M. 1990. Survey and discussion of the terminology used in bark anatomy. IAWA Bulletin n.s. 11: 141-166.

Trockenbrodt M, Parameswaran N. 1986. A contribution to the taxonomy of the genus Inga Scop. (Mimosaceae) based on the anatomy of the secondary phloem. IAWA Bulletin n.s. 7: 62-71.

Van Wyk AE. 1985. The genus Eugenia (Myrtaceae) in Southern Africa: structure and taxonomic value of bark. South African Journal of Botany 51: 157-180.

Venkaiah K, Shah JJ. 1984. Distribution, development and structure of gum ducts in Lannea coromandelica (Houtt.) Merril. Annals of Botany 54: 175-186.

Wendt T, Mitchell JD. 1995. A new species of Tapirira (Anacardiaceae) from the Isthmus of Tehuantepec, Mexico. Brittonia 71: 101-108.

Werker E, Fahn A. 1969. Resin ducts of Pinus halepensis Mill. -their structure, development and pattern of arrangement. Botanical Journal of the Linnean Society 62: 379-411.

Zahur MS. 1959. Comparative study of secondary phloem of 423 species of woody dicotyledons belonging to 85 families. Cornell Experiment Station Memoir 358: 4-259. 